TRANSACTIONS OF THE

AMERICAN MATHEMATICAL SOCIETY

Volume 172, October 1972

\title{
MATRIX RINGS OVER POLYNOMIAL IDENTITY RINGS
}

BY

\section{ELIZABETH BERMAN}

ABSTRACT. We prove that if $A$ is an algebra over a field with at least $k$ elements, and $A$ satisfies $x^{k}=0$, then $A_{n}$, the ring of $n$-by-n matrices over $A$, satisfies $x^{q}=0$, where $q=k n^{2}+1$. Theorem 1.3 generalizes this result to rings: If $A$ is a ring satisfying $x^{k}=0$, then for all $n$, there exists $q$ such that $A_{n}$ satisfies $x^{q}=0$.

Definitions. A checkered permutation of the first $n$ positive integers is a permutation of them sending even integers into even integers. The docile polynomial of degree $n$ is

$$
D\left(x_{1}, \cdots, x_{n}\right)=\sum_{f} x_{f(1)} \cdots x_{f(n)},
$$

where the sum is over all checkered permutations $f$ of the first $k$ positive integers. The docile product polynomial of degree $k, p$ is

$$
\prod_{i=1}^{p} D\left(x_{i 1}, \cdots, x_{i k}\right) u_{i},
$$

where the $x$ 's and $u$ 's are noncommuting variables. Theorem 2.1. Any polynomial identity algebra over a field of characteristic 0 satisfies a docile product polynomial identity. Theorem 2.2. If $A$ is a ring satisfying the docile product polynomial identity of degree $2 k, p$, and $n$ is a positive integer, and $q=2 k^{2} n^{2}+1$; then $A_{n}$ satisfies a product of $p$ standard identities, each of degree $q$.

Claudio Procesi and Lance Small proved that if $A$ is an algebra over a field satisfying a polynomial identity of degree $d$ then, for all $n, A_{n}$ satisfies some power of the standard identity of degree $2 n[d / 2]^{2}$, where $[d / 2]$ is the largest integer in $d / 2$ [3]. Independently, this writer obtained the same theorem for a commutative ring with unity satisfying a homogeneous polynomial identity with at least one coefficient of one [1, Theorem 8]. But neither theorem specifies the power. This paper discusses polynomial identities which determine a specific polynomial identity for $A_{n}$.

The abstract above defines the docile polynomial of degree $k$. The unitary polynomial of degree $k$ is $\Sigma_{f} x_{f(1)} \cdots x_{f(k)}$, where the sum is over all permutations $f$ of the integers 1 through $k$. In [1, Theorem 1], we showed that if $A$ is a ring satisfying the unitary identity of degree $k$, then $A_{n}$ satisfies the unitary identity of degree $k n^{2}+1$. In [1, Theorem 2], we showed that if $A$ satisfies the

Received by the editors July 1,1971 .

AMS (MOS) subject classifications (1970). Primary 16A38, 16A22, 16A42.

Key words and phrases. Polynomial identity rings, nil rings, matrix rings. 
docile identity of degree $2 k$, then $A_{n}$ satisfies the standard identity of degree $2 k^{2} n^{2}+1$. The proofs resemble the proofs of Theorems 1.1 and 2.2 below.

1. Nil rings. We quote an easy lemma from a previous paper.

Lemma 1.1. If $k$ and $q$ are positive integers; $S$, a set with $k$ elements; $B$, a set with $k(q-1)+1$ elements; and there is a function from $B$ into $S$; then at least $q$ elements of $B$ bave the same image in $S$ [1, Lemma 1].

Definition 1.1. Suppose that $R$ is a ring and $r_{1}, \cdots, r_{q}$ is a sequence of $q$ elements of $R$. To parentbesize the sequence into $j$ clumps is to insert $j$ pairs of adjacent, nonoverlapping parentheses. The subsequence within one pair of parentheses constitute a clump. The product of the enclosed elements in the order stated is the value of the clump.

Theorem 1.1. If $A$ is a ring satisfying $x^{n}=0$, then $A$ satisfies the unitary identity of degree $n$. If $A$ is an algebra over a field of characteristic 0 or $p>n$, and $A$ satisfies the unitary identity of degree $n$, then $A$ satisfies $x^{n}=0$.

Proof. We prove the first statement by linearizing $x^{n}$ in the usual way [2]. The second statement results from'substituting $x$ for $x_{i}$, for all $i$, in the unitary polynomial. We get the polynomial identity $n ! x^{n}=0$. Since $p$ does not divide $n !, A$ satisfies $x^{n}=0$.

Definition 1.2. Let $k$ be a positive integer. Let $\left\{f_{i}\right\}$ be the set of homogeneous components of the polynomial $\left(\sum_{i=1}^{k} x_{i}\right)^{k}$. We call a ring $A$ an $N$-ring of degree $k$ if all the $f_{i}$ vanish on $A$.

Some examples of $N$-rings follow.

1. If $A$ is an algebra over a field with at least $k$ elements, and $A$ is nil of index $k$, then $A$ is an $N$-ring of degree $k$.

Proof. A satisfies

$$
0=\left(\sum_{i=1}^{k} x_{i}\right)^{k}=x_{1}^{k}+\cdots+x_{k}^{k}+x_{1}^{k-1} x_{2}+\cdots
$$

The first $k$ terms on the right vanish on $A$. Using a Vandermonde determinant, we see that every homogeneous component vanishes on $A$. ,

2. If $A$ is an algebra over a field of characteristic 0 or $p>k$, and one of the homogeneous components of $\left(\sum_{i=1}^{k} x_{i}\right)^{k}$ vanishes on $A$, then $A$ is an $N$-ring of degree $k$.

Proof. Each component yields the unitary polynomial upon linearization. Now we use Theorem 1.1 and the Example 1.

3. If $A$ satisfies $x^{2}=0$, then $A$ is an $N$-ring of degree 2 . 
Theorem 1.2. If $A$ is an $N$-ring of degree $k$, then for all $n, A_{n}$ satisfies $x^{q}=0$, where $q=k n^{2}+1$.

Proof. Let $Z$ be the ring of the integers. Then $A_{n}$ is isomorphic to $A \otimes Z_{n}$. Let $u \in A \otimes Z_{n}$. Then $u$ is a sum of simple tensors:

$$
u=\sum_{i=1}^{m} c_{i} \otimes w_{i},
$$

where $c_{i} \in A$, and $w_{i}$ is a matrix unit.

$$
u^{q}=\sum_{f} c_{f(1)} \cdots c_{f(q)} \otimes w_{f(1)} \cdots w_{f(q)},
$$

where the sum is over all functions $f$ from $\{1, \cdots, q\}$ into $\{1, \cdots, m\}$.

Let $t_{f}$ be a term in this expansion. Consider the sequence of matrix units corresponding to the right side of $t_{f}$ :

$$
S=\left\{w_{f(1)}, \cdots, w_{f(q)}\right\} .
$$

By Lemma 1.1 , at least $k+1$ of these matrix units in $S$ are the same. Call them all $y$, the distinguished matrix unit of the term. Call the summands of $u$ appearing in $t_{f}$ :

$$
a_{1} \otimes y, a_{2} \otimes y_{1} \cdots, b_{1} \otimes z_{1}, b_{2} \otimes z_{2}, \cdots
$$

Then $S$ has form such as the following, where we have parenthesized into clumps.

$$
\left\{z_{1}, z_{1},\left(y, z_{2},\right)\left(y, z_{3},\right)(y,) \cdots, y, z_{4}\right\} \text {. }
$$

As in this example, we parenthesize $S$ into clumps as follows: start with the first $y$ and enclose it in parentheses along with all $z$ 's to the right, if any, up to the next $y$. Continue, forming $k$ clumps, each containing one $y$. There are at least $k+1 y$ 's, but just the first $k$ get parentheses. Now consider $f$ as a sequence: $\{f(1), \cdots, f(q)\}$. Parenthesize $f$ in the same manner as $S$, forming $k$ clumps.

Consider the sequence corresponding to the left side of $t_{f}: A=\left\{c_{f(1)}, \cdots, c_{f(q)}\right\}$. Parenthesize $A$ in the same manner as $S$ and $f:$ start with the first $a$ and enclose it in parentheses, along with all $b$ 's to the right, up to the next $a$. Continue, forming $k$ clumps.

We can partition into disjoint subsets the set of all functions from $q$ integers into $m$ integers, such that if $f$ and $f^{\prime}$ are in the same subset, they have the following relation:

(1) The entries before the first clump are the same entries in the same order.

(2) The entries after the last clump are the same in the same order.

(3) The $k$ clumps are the same, but in any order. If a clump appears exactly $j$ times in $f$, it appears exactly $j$ times in $f^{\prime}$. 
Let $T$ be one of the partition subsets so defined. Here is an example of two terms $t_{f}$ and $t_{f}^{\prime}$, such that $f$ and $f^{\prime}$ are both in $T$. We have inserted parentheses on both left and right sides, in the same manner as in the corresponding sequences. The sequences corresponding to the right sides have two distinct clumps, $\left(y, z_{1},\right)$ and $\left(y, z_{2},\right)$; the first appearing once; the second, $k-1$ times.

and

$$
\begin{gathered}
b_{1} b_{2}\left(a_{1} b_{1}\right)\left(a_{1} b_{2}\right)\left(a_{1} b_{2}\right) \cdots\left(a_{1} b_{2}\right) a_{3} b_{3} \cdots b_{p} \\
\otimes z_{1} z_{2}\left(y z_{1}\right)\left(y z_{2}\right)\left(y z_{2}\right) \cdots\left(y z_{2}\right) y z_{3} \cdots z_{p},
\end{gathered}
$$

$$
\begin{gathered}
b_{1} b_{2}\left(a_{1} b_{2}\right)\left(a_{1} b_{1}\right)\left(a_{1} b_{2}\right) \cdots\left(a_{1} b_{2}\right) a_{3} b_{3} \cdots b_{p} \\
\otimes z_{1} z_{2}\left(y z_{2}\right)\left(y z_{1}\right)\left(y z_{2}\right) \cdots\left(y z_{2}\right) y z_{3} \cdots z_{p} .
\end{gathered}
$$

We will show that the sum of all terms $t_{f} \ni f \in T$ is 0 . If $f \in T$, let the right side of the corresponding term be $w_{f}=w_{f(1)} \cdots w_{f(q)}$. If for all $f$ in $T$, $w_{f}=0$, then certainly the sum of all terms for $T$ is 0 . Suppose that for at least one $f^{\prime}$ in $T, w_{f}^{\prime} \neq 0$. We will show that for all $f$ in $T, w_{f}=w_{f}^{\prime}$. Let $e_{i j}$ be the matrix unit with the integer 1 in row $i$, column $j$. Let $S=\left\{w_{f^{\prime}(1)}, \cdots, w_{f^{\prime}(q)}\right\}$.

Case 1 . The sequence $S$ has two adjacent $y$ 's. Then there exists a matrix unit $e_{i i}$ such that $y=e_{i i}$. Hence each clump in $S$ must have value $e_{i i}$.

Case 2. Every clump in $S$ has at least one $z$. Let $y=e_{i j}$. Then in each clump, the product of the $z^{\prime}$ s must be $e_{j i}$, for otherwise $w_{f}^{\prime}=0$. Thus each clump in $S$ has value $e_{i i}$.

If $f$ and $f^{\prime}$ are both in $T$, then $w_{f}=w_{f}^{\prime}$, because the corresponding sequences of matrix units are alike except for a permutation of clumps.

Thus the sum of all terms corresponding to $f \in T$ is a simple tensor, whose right side is $w_{f}^{\prime}$. The left side is a product of factors:

(1) The product of all the $b$ 's before the first $a$.

(2) One of the $f_{i}$ from Definition 1.2, evaluated on the $k$ clumps containing a's.

(3) The product of all the entries after the last clump.

Since the second factor is 0 , the theorem follows.

We give an example of the second factor, using the two related terms mentioned above.

There were two distinct clumps for the left sides: $\left(a_{1}, b_{1},\right)$, and $\left(a_{1}, b_{2},\right)$; the first appearing once; the second, $k-1$ times. The appropriate polynomial is

$$
x_{1} x_{2}^{k-1}+x_{2} x_{1} x_{2}^{k-2}+\cdots+x_{2}^{k-1} x_{1} .
$$

The following theorem generalizes the above theorem to a matrix ring over any nil $r$ ing of bounded index, but does not yield a specific degree. 
Theorem 1.3. If $A$ is a ring satisfying $x^{k}=0$, then for all $n$ there exists $q$ such that $A_{n}$ satisfies $x^{q}=0$.

Proof. First, note that if $g$ is a homomorphism from ring $R$ into $R^{\prime}$, with $g(r)=r^{\prime}$, then $g$ induces a homomorphism $G$ of $R_{n}$ into $R_{n}^{\prime}: G\left[\left(r_{i j}\right)\right]=\left(r_{i j}^{\prime}\right)$. Clearly, $\operatorname{ker} G=(\operatorname{ker} g)_{n}$.

Let $B$ be the ring of polynomials with integral coefficients in $n^{2}$ noncommuting variables $\left\{e_{i j}\right\}, 1 \leq i, j \leq n$. Let $D$ be the ideal of $B$ generated by elements of form $b^{k}$ for some $b \in B$. Then $B / D$ satisfies $x^{k}=0$. Let $E$ be the matrix in $B_{n}$ whose entry in row $i$, column $j$, is $e_{i j}$. Let $E^{\prime}$ be the matrix in $(B / D)_{n}$ whose entry in row $i$, column $j$, is $e_{i j}^{\prime}$.

Since $B / D$ is bounded nil, it is locally nilpotent, and thus $(B / D)_{n}$ is also locally nilpotent. Hence there exists $q$ such that $E^{\prime} q=0$. Thus in $B_{n}, E^{q}=$ $\left(d_{i j}\right) \in D_{n}$.

Let $\left(a_{i j}\right) \in A_{n}$. Define a function $g$ : let $g\left(e_{i j}\right)=a_{i j}$, and extend $g$ to get a homomorphism $g$ from $B$ into $A$. Then $g$ induces a homomorphism $G$ from $B_{n}$ into $A_{n}$. Since $A$ satisfies $x^{k}=0, D$ lies in the kernel of $g$, and thus $D_{n}$ lies in the kernel of $G$. Obviously, $G(E)=\left(a_{i j}\right)$. Thus

$$
\left(a_{i j}\right)^{q}=[G(E)]^{q}=G\left(E^{q}\right)=G\left[\left(d_{i j}\right)\right]=0 .
$$

2. The docile product identity. We restate two results from a previous paper.

Lemma 2.1. If we have $n$ ordered elements of a set and insert parentheses to form $j$ clumps, each containing an odd number of elements, and permute these clumps with some clump permutation $f$, then the resulting permutation of the original elements is even if $f$ is even and odd if $f$ is odd [1, Corollary to Lemma 4].

Lemma 2.2. If $A$ is a ring and $A_{2}$ satisfies the standard identity of degree $k$, then $A$ satisfies the docile identity of degree $k$ [1, Theorem 3].

Lemma 2.3. Suppose that $n$ is a positive integer and $A$ is an algebra over $a$ field $F$ of characteristic 0 or $p>n$. Let $g\left(x_{1}, \ldots, x_{m}\right)$ be a polynomial over $F$ such that $A$ satisfies $\left[g\left(x_{1}, \cdots, x_{m}\right)\right]^{n}=0$. Then $A$ satisfies $\Pi_{i=1}^{N} g\left(x_{i 1}, \cdots, x_{m}\right)=0$, where $N=2^{n}-1$.

Proof. The proof is almost identical to the P. J. Higgins proof of the NagataHigman theorem: if $A$ is an algebra over a field of characteristic 0 or $p>n$, and $A$ satisfies $x^{n}=0$, and $N=2^{n}-1$, then $A$ satisfies $x_{1} x_{2} \cdots x_{N}=0[2$, p. 274].

Theorem 2.1. If $A$ is a polynomial identity algebra of degree $d$ over a field 
$F$ of characteristic 0 , and $k=4[d / 2]^{2}$, then there exists $j$ such that $A$ satisfies the docile product identity of degree $k, j$.

Proof. Let $A$ satisfy $f$ of degree $d$. Let $B$ be the free algebra over $F$, freely generated by $e_{1}, e_{2}, \ldots$. Let $D$ be the ideal of $B$ generated by elements of form $f\left(b_{1}, \cdots\right)$, where for all $i, b_{i} \in B$. Let $Q=B / D$. Let $N$ be the upper nil radical of $Q$. Then $Q / N$ has no nonzero nil ideals and satisfies $f$. Hence $(Q / N)_{2}$ satisfies the standard identity of degree $k=4[d / 2]^{2}[2$, p. 227]. Thus by Lemma 2.2, $Q / N$ satisfies the docile identity of degree $k: g\left(x_{1}, \cdots, x_{k}\right)=0$.

For all $i$, let $e_{i}^{\prime}=e_{i}+D \in Q$. Let $e_{i}^{\prime \prime}=e_{i}^{\prime}+N$. Then in $Q / N$ :

$$
g\left(e_{1}^{\prime \prime}, \cdots, e_{k}^{\prime \prime}\right) e_{k+1}^{\prime \prime}=0 \text {. }
$$

Thus in $Q$ :

$$
g\left(e_{1}^{\prime}, \cdots, e_{k}^{\prime}\right) e_{k+1}^{\prime}=w \in N .
$$

There exists $t>0$ such that $w^{t}=0$. Thus in $B$ :

$$
\left[g\left(e_{1}, \cdots, e_{k}\right) e_{k+1}\right]^{t}=d \in D .
$$

Now let $a_{1}, \cdots, a_{k+1}$ be any $k+1$ elements of $A$. If $1 \leq i \leq k+1$, let $b\left(e_{i}\right)=a_{i}$. Extend $b$ to get a homomorphism from $B$ into $A$. Let

$$
g^{\prime}\left(x_{1}, \cdots, x_{k}, u\right)=\left[g\left(x_{1}, \cdots, x_{k}\right)\right] u .
$$

Thus

$$
\begin{aligned}
& {\left[g^{\prime}\left(a_{1}, \cdots, a_{k}, a_{k+1}\right)\right]^{t}} \\
& \quad=\left[g^{\prime}\left(b\left(e_{1}\right), \cdots, b\left(e_{k}\right), b\left(e_{k+1}\right)\right)\right]^{t} \\
& \quad=b\left(\left[g^{\prime}\left(e_{1}, \cdots, e_{k}, e_{k+1}\right)\right]^{t}\right)=b(d)=0 .
\end{aligned}
$$

Thus $A$ satisfies the $t$ th power of $g^{\prime}$. By Lemma 2.3, A satisfies the docile product identity of degree $k, T$.

Let the standard polynomial of degree $q$ be the polynomial yielding the standard identity of degree $q$.

The conclusion of the following theorem says that $A_{n}$ satisfies a product of $p$ standard identities, each of degree $q$. It is interesting that $p$ does not depend on $n$.

Theorem 2.2. If $A$ is a ring satisfying the docile product identity of degree $k, p$, and $n$ is a positive integer, $q=2 k^{2} n^{2}+1$, and $g$ is the standard polynomial of degree $q$, then $A_{n}$ satisfies $0=\prod_{i=1}^{p} g\left(x_{i 1}, \cdots, x_{i q}\right)$.

Proof. Let $b$ be the polynomial on the right of the above identity. Now $A$ can be imbedded in a ring with unity [3, p. 87]. Both rings satisfy the docile 
product identity of degree $2 k, p$. Thus we assume that $A$ has a unity. Consider $A \otimes Z_{n}$, isomorphic to $A_{n}$. Choose $p \cdot q$ simple tensors in $A \otimes Z_{n}$ of form $c_{i} \otimes w_{i}$, where $c_{i} \in A$, and $w_{i}$ is a matrix unit. Since $b$ is multilinear and homogeneous, it suffices to prove that $b$ vanishes on such elements. Evaluating $b$, we get a sum of terms, each a simple tensor. The right side of each is a product of $p \cdot q$ matrix units. Call the sequence yielding the right side of a given term $\left\{w_{1}, \cdots, w_{p q}\right\}$. Section the above sequences into $p$ sections, each containing $q$ elements. Similarly section the sequence corresponding to the left side of each term.

In each section for the right side, there is a matrix unit that appears at least $2 k^{2}+1$ times, by Lemma 1.1. Call this matrix unit $y$, the distinguished matrix unit of the section. We can assume that for all $i$, section $i$ of every sequence has the same distinguished matrix unit. Fix $i$. Call the simple tensors whose product yields section $i$ :

$$
a_{1} \otimes y, a_{2} \otimes y, \cdots \quad \text { and } \quad b_{1} \otimes z_{1}, b_{2} \otimes z_{2}, \cdots
$$

Insert parentheses in each section for the right: start with the first $y$. Enclose it in parentheses, along with all $z$ 's to the right, if any, up to the next $y$. Continue, making $2 k^{2}$ pairs of parentheses. Similarly, make $2 k^{2}$ clumps on the sequence of $a$ 's and $b$ 's, enclosing each $a$ except the last with all $b$ 's to the right. Parenthesize each section this way.

A clump is odd or even depending on whether it has an odd or even number of elements. If a section contains $2 k$ or more odd clumps, call the section odd, and proceed as follows: introduce a new parenthesizing with brackets. Start with the first odd clump. Enclose it in brackets, along with all even clumps to the right, if any, up to the next odd clump. The bracketed elements for a super-clump, which contains an odd number of elements. Continue, so as to make $2 k$ superclumps. They form the distinguished subsection of the section .

If the number of odd clumps in the section is less than $2 k$, call the section even. Subdivide the section into $2 k$ subsections, each consisting of $k$ clumps. One subsection must consist entirely of even clumps, because if each subsection contained at least one odd clump, there would be at least $2 k$ odd clumps in the section. Call the first $k$ consecutive even clumps the distinguished subsection of the section. The b's to the right of each a form a sub-clump. In the distinguished subsection all the sub-clumps are odd.

We introduce an equivalence relation on the sequences corresponding to the left sides of the terms: we call sequence $W$ equivalent to sequence $W^{\prime}$ if the following hold for all $i$ :

(1) If $S_{i}$ is section $i$ of $W$; and $S_{i}^{\prime}$, of $W^{\prime}$; then either both $S_{i}$ and $S_{i}^{\prime}$ are even, or both are odd. The elements to the left of the distinguished subsection 
in $S_{i}$ and $S_{i}^{\prime}$ are the same elements in the same order. The elements to the right of the distinguished subsection are the same elements in the same order.

(2) If $S_{i}$ and $S_{i}^{\prime}$ are both odd, then the $2 k$ super-clumps are the same, in any order, so long as the arrangement in $S_{i}^{\prime}$ results from a checkered permutation of the arrangement in $S_{i}$. (The abstract defines checkered permutations.)

(3) If both $S_{i}$ and $S_{i}^{\prime}$ are even, then in the distinguished subsection the $k$ adjacent even clumps have the same $a$ 's and the same $b$ sub-clumps. We permit any order of the $a$ 's and $b$ sub-clumps, so long as they alternate, starting with an $a$.

For example, if $k=n=2$, then $2 k^{2} n^{2}+1=33$. There are $2 k^{2}+1=9$ a's in each section. We form 8 clumps in each section. Here is an example of two equivalent odd sections, with the distinguished subsections underlined:

$$
\begin{array}{r}
\left\{b_{1}, b_{2},\left(a_{1}, b_{3},\right)\left[\left(a_{2},\right)\left(a_{3}, b_{4},\right)\right]\left[\left(a_{5}, b_{6}, b_{7},\right)\right]\right. \\
\left.\left[\left(a_{6},\right)\right]\left[\left(a_{7}\right)\right]\left(a_{8}, b_{8},\right)\left(a_{4}\right), a_{9}, \cdots, b_{24}\right\}
\end{array}
$$

and

$$
\begin{aligned}
& \left\{b_{1}, b_{2},\left(a_{1}, b_{3},\right)\left[\left(a_{6},\right)\right]\left[\left(a_{7}\right)\right]\left[\left(a_{2},\right)\left(a_{3}, b_{4},\right)\right]\right. \\
& \left.\left[\left(a_{5}, b_{6}, b_{7},\right)\right]\left(a_{8}, b_{8},\right)\left(a_{4},\right) a_{9}, \cdots, b_{24}\right\} .
\end{aligned}
$$

Here are two equivalent even sections, with the distinguished subsections underlined:

and

$$
\begin{aligned}
& \left\{b_{1}, b_{2},\left(a_{1}, b_{3}, b_{4},\right) \frac{\left(a_{2}, b_{5},\right)\left(a_{3}, b_{6}, b_{7}, b_{8},\right)}{\left.\left(a_{4}, b_{9},\right)\left(a_{5}, b_{10},\right)\left(a_{6},\right)\left(a_{7},\right)\left(a_{8},\right) a_{9}, \cdots, b_{24}\right\}}\right.
\end{aligned}
$$

$$
\begin{aligned}
& \left\{b_{1}, b_{2},\left(a_{1}, b_{3}, b_{4},\right)\left(a_{2}, b_{6}, b_{7}, b_{8},\right)\left(a_{3}, b_{5},\right)\right. \\
& \left.\left(a_{4}, b_{9},\right)\left(a_{5}, b_{10},\right)\left(a_{6},\right)\left(a_{7},\right)\left(a_{8},\right) a_{9}, \cdots, b_{24}\right\} \text {. }
\end{aligned}
$$

Consider a fixed equivalence class. We will show that the sum of all the terms corresponding to the class is 0 . Without loss of generality, at least one term has right side nonzero. Then all the right sides under consideration are equal. From an odd section we get the docile polynomial of degree $2 k$, evaluated on the values of the $2 k$ odd super-clumps. From an even section we get the docile polynomial of degree $2 k$, evaluated on the $k a$ 's and the values of the $k$ sub-clumps of $b$ 's. The elements to the right of each distinguished subsection, up to the next distingu ished subsection, correspond to the $u$ 's in the docile product polynomial. If necessary, insert the unity from $A$ at the end of a section to get the $u$. The sum of all the terms for the class is thus a simple tensor whose right side is the common value of all the right sides, and whose left side is the product of the following:

(1) The product of all the elements in the first section left of its distinguished subsection. 
(2) The docile product polynomial of degree $2 k, p$, evaluated on the appropriate super-clumps, sub-clumps, and elements.

Since the second factor is $0, b$ vanishes on $A_{n}$.

\section{REFERENCES}

1. Elizabeth Berman, Tensor products of polynomial identity algebras, Trans. Amer. Math. Soc. 156 (1971), 259-271. MR 43 \#278.

2. Nathan Jacobson, Structure of rings, rev. ed., Amer. Math. Soc. Colloq. Publ., vol. 37, Amer. Math. Soc., Providence, R. I., 1964. MR 36 \#5158.

3. Neal H. McCoy, Rings and ideals, Carus Monograph Series, no. 8, Open Court, LaSalle, Ill., 1948. MR 10,96

4. Claudio Procesi and Lance Small, Endomorphism rings of modules over PI-algebras, Math. Z. 106 (1968), 178-180. MR 38 \#2167.

DEPARTMENT OF MATHEMATICS, ROCKHURST COLLEGE, KANSAS CITY, MISSOURI 64110 